\title{
Evaluation of the Implementation of a Rapid Response Treatment Protocol for Patients with Acute Onset Stroke: Can We Increase the Number of Patients Treated and Shorten the Time Needed?
}

\author{
Rajiv Advani ${ }^{a, b}$ Halvor Naess ${ }^{c}$ Martin W. Kurz ${ }^{a, b}$ \\ ${ }^{a}$ Department of Neurology and ${ }^{b}$ Neuroscience Research Group, Stavanger University \\ Hospital, Stavanger, and 'Department of Neurology, Haukeland University Hospital, \\ Bergen, Norway
}

Key Words

Rapid response treatment protocol $\cdot$ Acute onset ischemic stroke $\cdot$ Intravenous thrombolysis

\section{Abstract}

Aims: This study aims to evaluate the implementation of a rapid response treatment protocol for patients presenting with acute onset ischemic stroke. Improvements of routines surrounding the admission and treatment of patients with intravenous thrombolysis (IVT), such as door-to-needle (DTN) times, and increasing the numbers of patients treated are discussed. Methods: We conducted a retrospective analysis of all patients $(n=320)$ treated with IVT for acute onset ischemic stroke at the Stavanger University Hospital, Norway, between 2003 and 2012. In 2009, a succession of changes to pre- and intra-hospital routines were made as well as an improvement in the education of primary health care physicians, nurses and paramedics involved in the treatment of acute onset stroke patients (rapid response treatment protocol). Analyses of DTN times, onset-to-needle times and the number of patients treated per year were carried out to ascertain the effect of the changes made. The primary aim was to analyze DTN times to look for any changes, and the secondary aim was to analyze changes in the number of patients treated per year. Results: In the years after the implementation of the rapid treatment protocol, we saw an improvement in the median DTN time with a decrease from 73 to $50 \mathrm{~min}$ in the first year $(p=0.03)$, a decrease of $45 \mathrm{~min}$ in the second year $(p=0.01)$ and a decrease of $31 \mathrm{~min}$ in the third year $(p<0.001)$. Similarly, an improvement in the number of patients treated per year was seen after enhancements in the treatment chain were made. A significant, 27-fold increase was shown when the number of patients treated in 2012 was compared with all patients treated in all years prior to the implementation of the rapid treatment protocol. Conclusions: The implementation of the rapid treatment protocol for 
Advani et al.: Evaluation of the Implementation of a Rapid Response Treatment Protocol for Patients with Acute Onset Stroke

acute onset ischemic stroke patients led to a significant decrease in the DTN time at our center. These improvements also produced an increase in the number of patients treated per year. The extension of the therapeutic window from 3 to $4.5 \mathrm{~h}$ for the use of intravenous recombinant tissue plasminogen activator also played a role in the increased treatment numbers.

(C) 2014 S. Karger AG, Basel

\section{Introduction}

Stroke is the most frequent cause of permanent disability in the industrialized world and one of the most frequent causes of death [1,2]. Besides the deep personal consequences for the affected individuals, it also results in an enormous financial burden to society [3]. The therapeutic effect of intravenous thrombolysis (IVT) during the first hours of an acute onset ischemic stroke is well documented, and it is now well established as the first-line treatment modality [4, 5]. IVT clearly has the potential to abate the serious consequences of ischemic stroke, but its efficacy is very much time dependent. The number of patients needed to treat to achieve a good outcome (defined as modified Rankin Scale $<2$ ) is 4.5 if treatment is initiated within $1.5 \mathrm{~h}$. That number doubles to 9 if treatment is initiated between 1.5 and $3 \mathrm{~h}$ and increases further to 14 if treatment is administered between 3 and $4.5 \mathrm{~h}$. When expressed in neuronal loss/minute, a maximum of 1.9 million neurons and 14 billion synapses are lost if a large vessel stroke remains untreated [6]. These numbers clearly show that a streamlined pre- and intra-hospital treatment chain is a prerequisite for the successful therapy of acute onset ischemic stroke. In addition, these numbers suggest that even the smallest time-saving measures can be of enormous significance.

IVT was licensed for the therapy of acute onset ischemic stroke in Norway and Europe in 2003. It was in that same year that we began treating our first patients at the Stavanger University Hospital (SUS). However, it was in 2009 that we started working systematically to streamline the treatment chain for patients presenting with acute onset ischemic stroke, revising and improving the pre- and intra-hospital routines around the treatment of these patients. The primary aim of our study was to retrospectively evaluate the significance of these changes in the procedure with respect to the number of patients treated with IVT. The secondary aim of the study was to evaluate changes in the door-to-needle (DTN) and onsetto-needle (OTN) times resulting from the changes in pre- and intra-hospital routines.

\section{Materials and Methods}

\section{Study Population and Outcome Measures}

SUS serves a large catchment area with a population of more than 450,000 , receiving all ischemic stroke patients in this area. Approximately 220,000 inhabitants live in and around the city of Stavanger; the remainder live in more rural areas of Rogaland County.

All patients who received IVT at SUS in the period between 2003 and $2012(\mathrm{n}=320)$ were included in the study. According to the guidelines, IVT was administered within $3 \mathrm{~h}$ from symptom onset in the years 2003-2008, and from 2009 onward, the time window for treatment administration was extended to $4.5 \mathrm{~h}$ (2009-2012).

All patients admitted with ischemic stroke were identified through patient records at the hospital, and the ones who had been treated with IVT were retrospectively complied into a database. Demographical factors and stroke risk factors were collected from the patients' medical records. All applicable times, including the time of stroke onset, the time at which the emergency medical service (EMS) was contacted, the time of admission and the time of IVT 
Advani et al.: Evaluation of the Implementation of a Rapid Response Treatment Protocol for Patients with Acute Onset Stroke

administration, were taken from the prehospital records (AMIS - acute medical information system) and from the hospital records. The pertinent time durations of prehospital transport time, which was the time that elapsed from the patient calling the emergency medical services until he/she was in the emergency room (ER), DTN and OTN times were calculated. These were then compared year for year to ascertain any changes after the implementation of the rapid treatment protocol. By using the database, it was also possible to look for changes in the total number of patients treated with IVT.

\section{Prehospital and ER Structuring}

Rogaland County is served by an emergency medical service comprising road-going ambulances, ambulance boats and an air ambulance service. The EMS personnel are either trained paramedics and/or qualified nurses.

The ER is staffed continuously by an on-call registrar in neurology. There are an on-call consultant neurologist who can be contacted by telephone at any time and nurses who are randomly assigned to patients as they are admitted, triaging and assisting with every acute admission. A computed tomography (CT) scanner in very close proximity to the ER is on standby for $24 \mathrm{~h}$ a day and staffed by two radiographers and a registrar in radiology. There is also a consultant radiologist on call, who can be contacted to review CT scans if necessary.

\section{Implementation of the Rapid Treatment Protocol}

From 2003 to 2006, all patients with suspected acute onset ischemic stroke (onset $<3 \mathrm{~h}$ ) who had been examined and diagnosed by a primary health care physician/general practitioner to have neurologic deficit were transported to the hospital ER. However, these patients were not the highest priority in the ER; this was in principle due to the use of the Manchester triage system, which meant that they were seen as nonacute cases. The patients were then assessed by a neurology registrar and referred to a head CT. If indicated, IVT was administered, but this occurred at the neurointensive care unit, situated at a considerable distance from the ER.

After 2007, there was a change in the prehospital treatment cascade. Patients with suspected acute onset stroke symptoms were able to directly contact the EMS. If the patient complained of any FAST symptoms [3], an ambulance was sent to the patient's location for rapid assessment by paramedics. Provided that the paramedics confirmed the presence of FAST symptoms on clinical examination, the patient would be urgently transported to the ER. Despite this change in prehospital routine, patients were, as in previous years, down-prioritized once in the ER due to the use of the Manchester triage system.

From 2009 onward, we conducted annual reviews of stroke treatment routines at all departments participating in the management of acute stroke patients. After each review, a specialist stroke neurologist would lecture at each of the participating departments to better inform staff with the hope of improving routines year by year.

In addition, from 2009 onward, regular seminars were arranged to advise GPs and primary health care physicians not to assess patients with suspected acute onset stroke but to send them directly to the ER with the help of the EMS.

The EMS operator and the chief nurse in the ER had the role of coordinating both pre- and intra-hospital resources so that the on-call neurologist, the nurses, the radiologist and radiographers were all awaiting the arrival of the patient, thus minimizing any possible delays.

The triaging system in the ER was also changed in 2009. If patients with suspected acute stroke (FAST symptoms) were admitted, they would be seen as a high priority. Not only one but two nurses were assigned to each patient to ensure efficacious patient management. Blood samples were taken and an echocardiogram was made in the ER before the patients were transported to the CT scanner. 

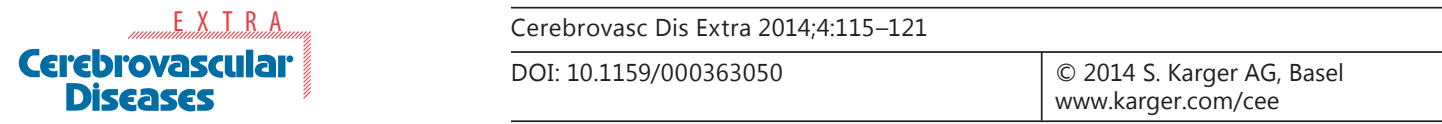

www.karger.com/cee

Advani et al.: Evaluation of the Implementation of a Rapid Response Treatment

Protocol for Patients with Acute Onset Stroke

Table 1. Number of patients treated with IVT per time interval and per year

\begin{tabular}{lllllllllll}
\hline & 2003 & 2004 & 2005 & 2006 & 2007 & 2008 & 2009 & 2010 & 2011 & 2012 \\
\hline Number of patients treated within $0-1.5 \mathrm{~h}$ & 0 & 0 & 0 & 0 & 0 & 0 & 5 & 12 & 30 & 25 \\
Number of patients treated within 1.5-3 h & 3 & 8 & 0 & 1 & 6 & 11 & 31 & 40 & 36 & 38 \\
Number of patients treated within $3-4.5 \mathrm{~h}$ & 0 & 0 & 0 & 0 & 0 & 2 & 9 & 22 & 20 & 21 \\
\hline Total number of patients treated $(\mathrm{n}=320)$ & 3 & 8 & 0 & 1 & 6 & 13 & 45 & 74 & 86 & 84 \\
\hline
\end{tabular}

If indicated, IVT treatment with recombinant tissue plasminogen activator was administered in the ER and not at the neurointensive care unit.

From 2012 onward, our intra-hospital routines were further streamlined. CT scanning was prioritized in patients presenting with acute onset stroke symptoms, meaning that the echocardiogram was taken after the initiation of IVT treatment. Additionally, patients who were not taking anticoagulants were treated with IVT immediately after the head CT, thus avoiding delays involved in the analysis of blood samples.

\section{Statistics}

Data analysis was conducted using the $\chi^{2}$ test for categorical variables, and one-way ANOVA was used for the comparison of continuous variables between the groups. A p value of $5 \%$ was considered significant. The study was approved by the local ethics committee.

\section{Results}

Table 1 shows the number of patients treated per year. In the years 2003-2007, we treated on average 3.6 patients per year (none in 2005). None of these patients was treated within $1.5 \mathrm{~h}$ from symptom onset. In 2008, after the patients were allowed to contact the EMS directly, the number of patients treated increased to $13 ; 2$ of them were treated within $1.5 \mathrm{~h}$ from symptom onset.

During the course of 2009, the rapid treatment protocol was implemented; the number of IVT treatments increased to 45 (36 of these treatments occurred within $3 \mathrm{~h}$ and 5 within $1.5 \mathrm{~h})$.

In 2010, the first complete calendar year with the rapid treatment protocol in effect, 74 patients were treated ( 52 within $3 \mathrm{~h}$ and 12 of them within $1.5 \mathrm{~h}$ ). The total number of patients treated increased to 86 and 84 in 2011 and 2012, respectively. In 2011, 66 of the 86 patients were treated within $3 \mathrm{~h}$ from symptom onset, while in 2012, the number was 63. In 2011, 30 patients were treated within $1.5 \mathrm{~h}$ from symptom onset compared to 25 in 2012 . We saw a statistically significant (27-fold) increase in treatments comparing the period between 2003 and 2008 with the year 2012. Only those patients treated within the 3-hour time window were compared. A further 21 patients were treated within the 3- to 4.5-hour time window in 2012. There was also a significant increase in the number of patients receiving treatment within the first $1.5 \mathrm{~h}$ from symptom onset when comparing the same years.

Table 2 shows the median DTN and OTN times for the years encompassed in our study. The median DTN time in the years 2003-2008 was $73 \mathrm{~min}$, decreasing to $50 \mathrm{~min}$ in 2010 $(p=0.03)$, which was the first complete calendar year with the rapid treatment protocol being in effect. The median DTN time further decreased from 73 min in the period between 2003 and 2008 to $45 \mathrm{~min}$ in 2011 ( $\mathrm{p}=0.01$ ). In 2012, with the established practice of IVT initiation 


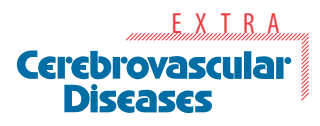

Table 2. Median DTN and OTN times (min) comparing the period before and after the implementation of the rapid treatment protocol

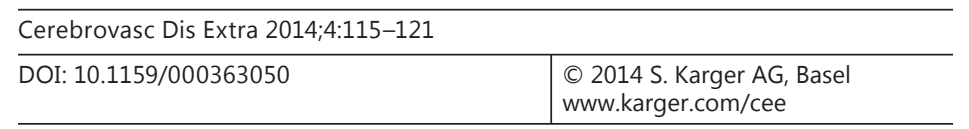

Advani et al.: Evaluation of the Implementation of a Rapid Response Treatment Protocol for Patients with Acute Onset Stroke

\begin{tabular}{lccl}
\hline & $2003-2008$ & $2010-2012$ & p value \\
\hline Median DTN time & 73 & 50 & 0.03 \\
Median OTN time & 135 & 134 & 0.74 \\
\hline & $2003-2008$ & 2011 & p value \\
\hline Median DTN time & 73 & 45 & 0.01 \\
Median OTN time & 135 & 122 & 0.67 \\
\hline & $2003-2008$ & 2012 & p value \\
\hline Median DTN time & 73 & 31 & $<0.001$ \\
Median OTN time & 135 & 119 & 0.44 \\
\hline
\end{tabular}

at the CT laboratory, the median DTN time was further reduced to $31 \mathrm{~min}$, with a p value $<0.001$ when compared to the period between 2003 and 2008.

The OTN time in the period between 2003 and 2008 was $135 \mathrm{~min}$. This was unchanged when compared to the OTN time in 2010 (134 min, $\mathrm{p}=0.74$ ). When compared to the OTN time in 2011 and 2012, we saw a modest reduction to 122 and $119 \mathrm{~min}$, respectively $(\mathrm{p}=0.66$ and $\mathrm{p}=0.44$ ).

Comparing the prehospital transport time before (2003-2008) and after (2009-2012) the implementation of the rapid treatment protocol showed no statistically significant change ( 40 and $48 \mathrm{~min}$, respectively, $\mathrm{p}=0.001$ ).

\section{Discussion}

The results of our study show that the implementation of the rapid treatment protocol led to a significant increase in the number of patients treated with IVT. There was an increase in each treatment window subset with the most significant increase occurring in the group of patients treated within $1.5 \mathrm{~h}$ from symptom onset. This increment in the first treatment window subset (treatment administered with $1.5 \mathrm{~h}$ from symptom onset) is most likely due to the reduction achieved in the DTN time (our secondary end point).

The statistically significant reduction in the DTN time gave us a time saving of $42 \mathrm{~min} /$ patient when median times were compared (2012 compared with 2003-2008).

The nature of retrospective data collection and analysis subjects the study to certain unavoidable weaknesses as there usually is an information bias and data collection is suboptimal in such studies. In this setting, a prospective cohort study would have facilitated the collection and analysis of the variables. The smaller subset of patients in the first years of our study gives a somewhat weaker quality of data, but also reflects the difficulty in the administration of IVT in the earlier years.

In 2000, the Brain Attack Coalition published recommended guidelines for the treatment of acute onset ischemic stroke, the target being a DTN time of no more than 60 min [7]. However, Fonarow et al. [8] found that of the 25,504 patients treated in the 641 participating centers in their study, $73.4 \%$ of them were not treated within the targeted 60 -min DTN time window.

Furthermore, the authors stressed the importance of reducing the DTN time. They found that each 15 -min reduction in the DTN time directly resulted in a 5\% lower chance of in-hospital mortality. In their patient population of over 25,000 , less than one third $(27 \%)$ 
Advani et al.: Evaluation of the Implementation of a Rapid Response Treatment Protocol for Patients with Acute Onset Stroke

had a DTN time of $<60$ min [8], further stressing the significance of a median DTN time of 31 min achieved at our hospital. This reduction in 2012 was achieved by a major change in the protocol, namely that of not waiting for blood work analysis but starting thrombolysis directly in the CT laboratory.

This practice involves a certain, quite significant risk that patients with thrombocytopenia or an elevated international normalized ratio could be treated with recombinant tissue plasminogen activator. In practice, this is only done in patients with an up-to-date preexisting medical record at SUS and in those whose earlier blood work showed normal results. This minimizes the risk of hemorrhage as a complication considerably when treating such patients.

Setting up a point-of-care laboratory in the ER for blood work analysis could help start thrombolysis earlier in all patients, even in those taking warfarin or those with an unknown previous medical history.

Fonarow et al. [9] also found that patients who had shorter OTN times were often those with DTN times $>60 \mathrm{~min}$, suggesting that hospitals took a relaxed approach to the treatment of these patients. Having a dedicated team of doctors and nurses working together with the implementation of the rapid treatment protocol seems to be pivotal in increasing the focus on the time elapsed after the onset of ischemic stroke. The authors suggest that the implementation of a revised treatment protocol would lead to a shortening of the DTN time for acute onset stroke patients.

The time dedicated to the neurologic assessment of acute stroke patients in the ER is crucial. A current review concluded that very few neurologists were used to working under the time pressure governing in the ER [10]. Therefore, most neurologists had to shift their focus to a more rapid patient assessment. This shift in focus, which is, to a certain extent, a change in the examination style, is one of the factors we believe played a role in the delayed improvements in the DTN times at SUS. The greatest changes in the DTN times coming 2 and 3 years after the most significant steps of the rapid treatment protocol had been implemented.

In 2012, progressive changes in the IVT treatment protocol implemented at the Helsinki University Hospital led to a significant reduction in the DTN time over a 10-year period. They achieved a median DTN time of only $20 \mathrm{~min}$, with $94 \%$ of their patients receiving IVT treatment within $60 \mathrm{~min}$ [11]. The groundbreaking strategies they applied completely circumvented the ER, taking patients directly from EMS transport to the CT scanner. This single change contributed to a 45-min reduction in the OTN time.

We have not yet implemented such drastic changes at SUS, yet in the 4 years since we started to change the way in which IVT treatment is administered, we have achieved a significant reduction in the DTN time. However, it is plain to see that taking patients directly to the CT scanner or bringing the CT scanner to the patients with specialized stroke ambulances can produce substantial time savings [12]. The medical benefit and safety of this innovative approach is still to be proven in clinical trials.

The median OTN time in our study shows some variation over the years (119-167 min). Although there was a downward trend in the years 2010, 2011 and 2012 (134, 122 and 119 min, respectively), there is no statistically significant reduction. This can, in some cases, be attributed to the geography of Rogaland County, with longer transport times for patients living in more remote areas. However, the primary source of delay seems to be the one occurring before the EMS is contacted by a stroke victim or a stroke witness [13]. This delay has been the focus of stroke information campaigns worldwide. Such information campaigns have shown variable effects, but no such campaigns were run at the time when the rapid treatment protocol was implemented at treatment centers. We plan to run a stroke information campaign during the course of the next year and analyze the effect it has on OTN and DTN times at our center at which the rapid treatment protocol is already in effect. 
The battle to reduce treatment times for patients presenting with acute onset ischemic stroke is an ongoing one. We demonstrated that the implementation of the rapid treatment protocol produces significant results in the process of achieving that goal. The measures each individual hospital can take to reduce these times are very much dependent on the local resources and the setup of acute medical care in that country, state and district. As shown by our study and others such as the one by Meretoja et al. [11], progressive changes are a fine step forward, and reductions in DTN times cannot be expected overnight. It takes practice and the implementation of a routine to achieve sustained results.

\section{References}

$>1$ Johnston SC, Mendis S, Mathers CD: Global variation in stroke burden and mortality: estimates from monitoring, surveillance, and modelling. Lancet Neurol 2009;8:345-354.

2 Rothwell PM, Coull AJ, Silver LE, et al: Population-based study of event-rate, incidence, case fatality, and mortality for all acute vascular events in all arterial territories (Oxford Vascular Study). Lancet 2005;366: 1773-1783.

-3 Olesen J, Gustavsson A, Svensson M, et al: The economic cost of brain disorders in Europe. Eur J Neurol 2012; 19:155-162.

-4 Tissue plasminogen activator for acute ischemic stroke. The National Institute of Neurological Disorders and Stroke rt-PA Stroke Study Group. N Engl J Med 1995;333:1581-1587.

5 Hacke W, Kaste M, Bluhmki E, et al: Thrombolysis with alteplase 3 to $4.5 \mathrm{~h}$ after acute ischemic stroke. $\mathrm{N}$ Engl J Med 2008;359:1317-1329.

6 Khatri P, Abruzzo T, Yeatts SD, et al: Good clinical outcome after ischemic stroke with successful revascularization is time-dependent. Neurology 2009;73:1066-1072.

7 Alberts MJ, Hademenos G, Latchaw RE, et al: Recommendations for the establishment of primary stroke centers. Brain Attack Coalition. JAMA 2000;283:3102-3109.

8 Fonarow GC, Smith EE, Saver JL, et al: Timeliness of tissue-type plasminogen activator therapy in acute ischemic stroke: patient characteristics, hospital factors, and outcomes associated with door-to-needle times within 60 min. Circulation 2011;123:750-758.

-9 Fonarow GC, Smith EE, Saver JL, et al: Improving door-to-needle times in acute ischemic stroke: the design and rationale for the American Heart Association/American Stroke Association's Target: Stroke initiative. Stroke 2011;42:2983-2989.

10 Lyden P: Thrombolytic therapy for acute stroke - not a moment to lose. N Engl J Med 2008;359:1393-1395.

11 Meretoja A, Strbian D, Mustanoja S, et al: Reducing in-hospital delay to 20 minutes in stroke thrombolysis. Neurology 2012;79:306-313.

12 Walter S, Kostopoulos P, Haass A, et al: Diagnosis and treatment of patients with stroke in a mobile stroke unit versus in hospital: a randomised controlled trial. Lancet Neurol 2012;11:397-404.

13 Kurz MW, Kurz KD, Farbu E: Acute ischemic stroke - from symptom recognition to thrombolysis. Acta Neurol Scand Suppl 2013:57-64. 